\title{
Ảnh hưởng của việc sử dụng phương tiện truyền thông điện tử lên thành quả công việc trong các đội gia công phần mềm từ xa \\ - Một khảo sát tại Việt Nam \\ The influence of electronic communication media on job performance of remote software outsourcing teams - A study in Vietnam
}

\author{
Huỳnh Thị Minh Châu ${ }^{1}$, Nguyễn Mạnh Tuân ${ }^{1}$, Trương Thị Lan Anh ${ }^{1}$ \\ ${ }^{1}$ Trường Đại học Bách Khoa, Đại học Quốc gia TP.HCM, Việt Nam \\ *Tác giả liên hệ, Email: htmchau@ hcmut.edu.vn
}

\begin{abstract}
THÔNG TIN
TÓM TÁT

DOI: $10.46223 /$ HCMCOUJS. econ.vi.14.3.482.2019

Ngày nhận: 06/06/2019

Ngày nhận lại: 30/07/2019

Duyệt đăng: 01/08/2019

Tù khóa:

đội ảo, gia công phần mềm từ xa, phương tiện truyền thông điện tử, thành quả công việc, tiếp tục sử dụng công nghệ thông tin

Ngoài các ứng dụng công nghệ thông tin phục vụ cho việc phát triển sản phẩm phần mềm, các đội gia công phần mềm từ xa còn sử dụng phương tiện truyền thông điện tử để giao tiếp và phối hợp làm việc trong đội. Bài báo này (1) đề xuất một mô hình cấu trúc về ảnh hưởng của bốn yếu tố liên quan đến việc sử dụng phương tiện truyền thông điện tử (gồm sự hài lòng với việc sử dụng, thói quen sử dụng, ý định tiếp tục sử dụng, mức độ sử dụng) lên thành quả công việc của thành viên đội gia công phần mềm từ xa, (2) tiến hành khảo sát định lượng trên 243 cá nhân đang là thành viên các đội gia công phần mềm từ xa tại Việt Nam. Kết quả cho thấy (i) sự hài lòng với việc sử dụng và thói quen sử dụng có ảnh hưởng tích cực lên ý định tiếp tục sử dụng phương tiện truyền thông điện tử, (ii) sự hài lòng với việc sử dụng và ý định tiếp tục sử dụng có ảnh hưởng tích cực lên mức độ sử dụng phương tiện truyền thông điện tử, (iii) mức độ sử dụng phương tiện truyền thông điện tử có ảnh hưởng tích cực lên thành quả công việc. Bốn yếu tố liên quan đến việc sử dụng phương tiện truyền thông điện tử nói trên giải thích được $16 \%$ sự biến thiên thành quả công việc.
\end{abstract}

\section{ABSTRACT}

In addition to information technology applications used for software development, remote software outsourcing teams also use electronic communication media for communication and coordination between each other. This paper (1) proposes a structural model describing the effects of four factors related to electronic communication media usage (including satisfaction with prior use, usage habit, IT continuance intention, level of usage) on 
Keywords:

electronic communication media, job performance, remote software outsourcing, information technology continuance, virtual team the job performance of remote software outsourcing team members; (2) conducts a quantitative survey on 243 members of remote software outsourcing teams in Vietnam. The results show that (i) satisfaction with prior use and usage habit have positive effects on continuance intention of using electronic communication media, (ii) satisfaction with prior use and continuance intention of using electronic communication media have positive effects on level of electronic communication media usage, (iii) level of electronic communication media usage has a positive effect on job performance. Four factors related to electronic communication media usage can explain $16 \%$ of variations on job performance.

\section{1. Đặt vấn đề}

Hình thức phát triển phần mềm từ xa được sử dụng phổ biến bởi các doanh nghiệp thuộc các nước phát triển, trong đó, trách nhiệm gia công phần mềm (GCPM) được ủy thác cho các nhà cung cấp đặt trụ sở tại Ân Độ, Ireland, Israel, Việt Nam... (Sahay, Nicholson, \& Krishna, 2003). Các nhà cung cấp này sở hữu lực lượng nhân sự có kiến thức chuyên môn, và có lợi thế chi phí đáng kể (Davis, Ein-Dor, King, \& Torkzadeh, 2006; Robinson \& Kalakota, 2004). Theo Forbes (Tran Ngoc, 2018), Việt Nam hiện đang nổi lên như một trung tâm GCPM đầy tiềm năng, ngành GCPM Việt Nam tăng trưởng đều đặn, vào năm 2017 Việt Nam đã tiến 5 bậc để xếp vị trí thứ 6 về GCPM toàn cầu. Theo công bố của Bộ Thông tin và Truyền thông Việt Nam (Van Anh, 2019), Việt Nam đang có khoảng 10.000 doanh nghiệp GCPM, nhân lực toàn ngành khoảng 120.000 người, vào năm 2018 ngành GCPM Việt Nam tăng trưởng 13,8\%, doanh thu ước đạt 4,3 tỷ USD, xuất khẩu ước đạt 3,5 tỷ USD.

Đội ảo là một cách sắp xếp công việc mà thành viên phân tán địa lý, hạn chế tiếp xúc trực tiếp và làm việc phụ thuộc lẫn nhau thông qua phương tiện truyền thông điện tử (PTTTĐT) nhằm đạt mục tiêu chung (Dulebohn \& Hoch, 2017). Đội ảo có nhiều lợi thế hơn đội truyền thống vì giúp các thành viên cộng tác xuyên qua giới hạn thời gian/không gian, giúp các tổ chức sử dụng tốt nguồn nhân lực bị phân tán mà không cần di chuyển về mặt vật lý (Friedrich, 2017). Các đội GCPM từ xa chủ yếu tổ chức công việc theo đội ảo (Huynh, Nguyen, \& Hang, 2017). Ngoài các ứng dụng công nghệ thông tin (CNTT) phục vụ cho việc phát triển sản phẩm phần mềm, các đội GCPM từ xa cũng sử dụng các PTTTĐT để phục vụ cho việc giao tiếp và cộng tác. PTTTĐT được đề cập trong các tài liệu bằng nhiều thuật ngữ khác nhau, như "công nghệ cộng tác" (Santillan \& Horwitz, 2016; Solomon, 2016), "công nghệ cộng tác và truyền thông/giao tiếp điện tử", "CNTT và truyền thông/giao tiếp" (Dube \& Marnewick, 2016), "công nghệ truyền thông/giao tiếp thông qua máy tính trung gian" (Lipnack \& Stamps, 2000), "công nghệ truyền thông/giao tiếp" (Chaves et al., 2016), "công nghệ ảo" (Greer, Luethge, \& Robinson, 2017), "CNTT" (Griffith, Sawyer, \& Neale, 2003) hay "công nghệ" nói chung (Wise, 2016)... Nhiều bằng chứng cho thấy trong đội ảo, việc tương tác gián tiếp thông qua công nghệ sẽ dẫn đến nhiều hệ lụy về mặt xã hội như sự hiểu lầm, tổn thương, xung đột, tranh giành quyền lực, thiếu tin cậy... (Ebrahim, Ahmed, \& Taha, 2011; Kayworth \& Leidner, 2002; Ocker \& Fjermestad, 2008). Bên cạnh đó, đôi khi đội ảo đòi hỏi ứng dụng PTTTĐT phức tạp (Bergiel, Bergiel, \& Balsmeier, 2008), không phù hợp thói quen, không làm thành viên đội hài lòng, vì 
vậy họ không có ý định sử dụng, không chấp nhận ngay từ đầu hoặc giảm sử dụng theo thời gian (Godin et al., 2017). Ozcelik (2010) cho rằng nếu nhà quản lý không cung cấp cơ chế hỗ trợ đầy đủ, khi việc sử dụng công nghệ gặp sự cố, các thành viên đội ảo có thể phải ngừng việc.

Bên cạnh đó, theo Bhattacherjee (2001), Bhattacherjee, Perols, và Sanford (2008), Bhattacherjee và Lin (2015), mặc dù sự chấp nhận ban đầu là tiền đề để CNTT/hệ thống thông tin (HTTT) được hiện thực hóa, nhưng việc tiếp tục sử dụng là quan trọng, vì sự tồn tại lâu dài và thành công của $\mathrm{CNTT/HTTT} \mathrm{phụ} \mathrm{thuộc} \mathrm{vào} \mathrm{việc} \mathrm{sử} \mathrm{dụng} \mathrm{liên} \mathrm{tục} \mathrm{chứ} \mathrm{không} \mathrm{phải} \mathrm{là} \mathrm{chấp}$ nhận sử dụng lần đầu. Việc nhân viên sử dụng HTTT không lâu dài, không thường xuyên, không thích hợp và không hiệu quả sẽ gây thất bại cho doanh nghiệp (Bhattacherjee, 2001). Việc duy trì người dùng CNTT hiện tại sẽ tiết kiệm hơn nhiều so với tìm người dùng mới (Bhattacherjee et al., 2008). Bhattacherjee (2001) chỉ ra những hạn chế khi sử dụng các tiền tố giải thích quyết định chấp nhận ban đầu để giải thích các quyết định sau khi sử dụng, và đề xuất mô hình tiếp tục sử dụng HTTT mô tả ảnh hưởng của sự hài lòng với việc sử dụng lên ý định tiếp tục sử dụng HTTT. Sau đó, Bhattacherjee et al. (2008) mở rộng nghiên cứu thêm hành vi tiếp tục sử dụng CNTT. Gần đây, Bhattacherjee và Lin (2015) thống nhất một mô hình tiếp tục sử dụng CNTT chứa 03 yếu tố trực tiếp giải thích hành vi tiếp tục sử dụng CNTT là: (1) sự hài lòng, (2) thói quen sử dụng, (3) ý định tiếp tục sử dụng. Theo Bhattacherjee và Lin (2015), sự hài lòng là một phản ứng tình cảm xuất phát từ kinh nghiệm sử dụng trước đó và có thể được xem là phản hồi mang tính trải nghiệm đối với việc sử dụng; thói quen là một chuỗi hành động mang tính học tập, hình thành có ý thức và được lặp lại một cách không có ý thức khi bị kích hoạt bởi môi trường; ý định tiếp tục sử dụng là dự định có ý thức hướng tới hành vi tiếp tục sử dụng.

Câu hỏi đặt ra là trong bối cảnh làm việc gián tiếp thông qua sự hỗ trợ của công nghệ như đội GCPM từ xa, các yếu tố liên quan đến việc sử dụng công nghệ hỗ trợ có ảnh hưởng như thế nào đến kết quả làm việc của thành viên đội? Bài báo này được thực hiện nhằm tìm hiểu cách thức ảnh hưởng của sự hài lòng với việc sử dụng PTTTĐT, thói quen sử dụng PTTTĐT, ý định tiếp tục sử dụng PTTTĐT và mức độ sử dụng PTTTĐT lên thành quả công việc của thành viên đội GCPM từ xa.

\section{Mô hình nghiên cứu}

Trong đội GCPM từ xa, mức độ sử dụng PTTTĐT là một hành động mang tính liên tục và đến sau sự chấp nhận ban đầu, nên dựa trên mô hình tiếp tục sử dụng CNTT của Bhattacherjee và Lin (2015), bài báo này đề xuất một mô hình cấu trúc mô tả cách thức ảnh hưởng của sự hài lòng với việc sử dụng PTTTĐT, thói quen sử dụng PTTTĐT, ý định tiếp tục sử dụng PTTTĐT và mức độ sử dụng PTTTĐT lên thành quả công việc của thành viên đội GCPM từ xa (xem Hình 1 - Mô hình sau khi đã kiểm định).

Dựa trên lý thuyết xác nhận kỳ vọng, Bhattacherjee (2001) biện luận rằng trong bối cảnh tiếp tục sử dụng HTTT, sự hài lòng với việc sử dụng trước đó là tiền đề của việc tiếp tục sử dụng vì người dùng có xu hướng tin tưởng nhiều vào trải nghiệm thực tế của họ hơn là mong đợi vào một tương lai chưa chắc chắn. Nhiều nghiên cứu thực nghiệm sau đó cũng chỉ ra rằng sự hài lòng ảnh hưởng đến hành vi tiếp tục sử dụng CNTT thông qua ý định của người dùng, và chứng minh rằng người dùng có ý định tiếp tục sử dụng một CNTT nhất định nếu họ có cảm xúc tích cực về việc sử dụng trước đó. Nếu không hài lòng, họ có xu hướng ngừng sử dụng và/hoặc chuyển sang CNTT/HTTT thay thế (e.g., Case, Cuellar, \& Tabatabaei, 2015; Gilani, Iranmanesh, Nikbin, \& Zailani, 2016; Ng, Ching, Chung, \& Dee, 2016; Piguing \& Ko, 2016; 
Shiue \& Hsu, 2017). Vì vậy, có căn cứ để đề xuất giả thuyết (H1): Sụ hài lòng với việc sủ dụng PTTTĐT có ảnh hương tích cưc lên ý định tiếp tục sử dụng PTTTĐT của thành viên đội GCPM tù $x a$.

Trong khi đó, một số nhà nghiên cứu khác lại cho rằng sự hài lòng có thể có ảnh hưởng trực tiếp lên hành vi mà không nhất thiết thông qua ý định (De Guinea \& Markus, 2009; Kim \& Malhotra, 2005; Scheier \& Carver, 1982). Scheier và Carver (1982) lưu ý rằng khi cảm xúc mâu thuẫn với ý định sẽ gây gián đoạn quá trình xử lý và sắp xếp lại các mục tiêu ưu tiên, nếu cảm xúc đủ mãnh liệt sẽ điều chỉnh được cả tổng thể, và khi sự chú ý của con người ở mức thấp thì con người thường cư xử theo cảm xúc. Kim và Malhotra (2005) gợi ý rằng nếu kết quả tiềm năng của việc sử dụng CNTT chưa được biết đầy đủ trước khi sử dụng, hành vi chấp nhận CNTT sẽ được thúc đẩy bởi ý định, nhưng trong trường hợp tiếp tục sử dụng, khi liên kết giữa các kích thích và hành động đã được thiết lập, người dùng ít đầu tư vào nhận thức mà thường dựa vào các phản ứng tình cảm, chẳng hạn như sự hài lòng. De Guinea và Markus (2009) lập luận là theo các nghiên cứu tâm lý học, mối liên hệ giữa cảm xúc và hành vi có thể xảy ra mà con người không hề có ý thức trước, cảm xúc có thể không tạo ra một ý định cụ thể hay làm hỏng một ý định có trước về việc tiếp tục sử dụng CNTT, và những cảm xúc như sự hài lòng có thể thúc đẩy việc sử dụng CNTT trực tiếp mà không nhất thiết phải thông qua trung gian là ý định. Một số nghiên cứu thực nghiệm cũng chứng minh sự hài lòng có thể có ảnh hưởng trực tiếp lẫn gián tiếp tới hành vi tiếp tục sử dụng CNTT (e.g., Bhattacherjee \& Lin, 2015; Doong \& Lai, 2008). Vì vậy, có căn cứ để đề xuất giả thuyết (H2): Sụ hài lòng với việc sủ dụng PTTTĐT có ảnh hường tích cưc lên mức độ sử dụng PTTTĐT của thành viên đội GCPM tù xa.

Bên cạnh đó, De Guinea và Markus (2009) tranh luận rằng việc một CNTT nhất định vốn đã được sử dụng trước đây được tiếp tục sử dụng để giải quyết một nhiệm vụ cụ thể có thể là bằng chứng cho việc tiếp tục sử dụng dựa trên thói quen. Mặc dù thói quen được cho là có ảnh hưởng đến việc tiếp tục sử dụng CNTT, con đường ảnh hưởng này không rõ ràng, một số nghiên cứu cho thấy thói quen có thể ảnh hưởng trực tiếp đến hành vi tiếp tục sử dụng CNTT (e.g., Kim \& Malhotra, 2005; Limayem \& Hirt, 2003), một số khác cho rằng phải thông qua trung gian là ý định tiếp tục sử dụng (e.g., Gefen, 2003). Vai trò tương đối của các phản ứng theo thói quen ở các giai đoạn khác nhau của quá trình sử dụng CNTT được xây dựng bởi Jasperson, Carter, và Zmud (2005). Theo đó, trong giai đoạn sử dụng ban đầu, các cá nhân có thể tham gia vào quá trình nhận thức để xây dựng ý định, tuy nhiên, đối với các hành vi mang tính lặp lại như một thói quen, phản xạ nhận thức tiêu tan theo thời gian, khi đó thói quen trực tiếp ảnh hưởng đến hành vi (Bhattacherjee \& Lin, 2015). Theo Wood, Quinn, và Kashy (2002), những người hành động theo thói quen không cần truy cập đến ý định, theo De Guinea và Markus (2009), khi hành vi trở thành thói quen thì có thể lặp đi lặp lại không cần ý định. Ảnh hưởng của thói quen sử dụng lên hành vi sử dụng có thể được xem là một sự phản biện cho quan điểm hành động hợp lý. Vì vậy, có căn cứ để đề xuất giả thuyết $(\boldsymbol{H 3})$ : Thói quen sủ dụng PTTTĐT có ảnh hương tích cưc lên ý định tiếp tục sủ dụng PTTTĐT của thành viên đội GCPM tù $x a$, và giả thuyết (H4): Thói quen sử dụng PTTTĐT có ảnh hưởng tích cực lên mức độ sư dụng PTTTĐT của thành viên đội GCPM tù xa.

Các lý thuyết tâm lý xã hội như TRA, TPB và các mô hình chấp nhận công nghệ như TAM, UTAUT từ lâu đã cho rằng hành vi cá nhân là có lý do và có kế hoạch, xuất phát từ ý định có ý thức liên quan đến hành vi đó. Nhiều nghiên cứu thực nghiệm được thực hiện trong bối cảnh sử dụng CNTT/HTTT, xác nhận rằng ý định sử dụng CNTT/HTTT của người dùng là yếu tố có ảnh hưởng lớn nhất đến hành vi sử dụng CNTT/HTTT (e.g., Agudo-Peregrina, 
Hernández-García, và Pascual-Miguel, 2014; Lian, Yen, \& Wang, 2014; Sun, Bhattacherjee, \& Ma, 2009; Thakur, Summey, \& John, 2013). Trong bối cảnh tiếp tục sử dụng CNTT/HTTT, nhiều nghiên cứu thực nghiệm cũng đã chứng minh người dùng có nhiều khả năng tiếp tục sử dụng một CNTT/HTTT nhất định nếu họ có ý định tích cực về việc tiếp tục (e.g., Huang, Wu, \& Chou, 2013; Kim, 2012; Limayem \& Cheung, 2008; Shanmugam, Jusoh, Nor, \& Jabar, 2015). Vì vậy, có căn cứ để đề xuất giả thuyết (H5): Ý định tiếp tục sử dụng PTTTĐT có ảnh hưởng tích cưc lên mức độ sủ dụng PTTTĐT của thành viên đội GCPM tù xa.

Sau cùng, để giải thích tác động của việc sử dụng PTTTĐT lên kết quả làm việc của thành viên trong đội GCPM từ xa, thành quả công việc được đưa vào mô hình như là một hậu tố của mức độ sử dụng PTTTĐT. Một số nhà nghiên cứu đã chỉ ra việc sử dụng công nghệ quyết định các kết quả làm việc cá nhân, đặc biệt là thành quả cá nhân (Burton-Jones \& Gallivan, 2007; Burton-Jones \& Straub, 2006; Sun et al., 2009). Tương tự, Jasperson et al. (2005) xác định cách sử dụng sau khi chấp nhận cài đặt các ứng dụng CNTT (như áp dụng các tính năng, mở rộng các tính năng...) giúp người dùng hoàn thành công việc, tạo ra các tác động tích lũy lên thành quả làm việc của hệ thống, thành quả công việc của người dùng được cải thiện khi họ sử dụng nhiều tính năng hơn và tìm ra cách sử dụng tính năng mới trong công việc. Vì vậy, có căn cứ để đề xuất giả thuyết (H6): Mức độ sử dụng PTTTĐT có ảnh hưởng tích cực lên thành quả công việc của thành viên đội GCPM tù xa.

\section{Phương pháp nghiên cứu}

Bảng câu hỏi gồm 02 biến nhân khẩu (Vị trí trong đội, Số thành viên của đội) và 24 biến đo lường kế thừa từ các nghiên cứu đi trước rồi hiệu chỉnh bằng phỏng vấn sơ bộ cho phù hợp với bối cảnh đội GCPM từ xa tại Việt Nam (xem Bảng 1).

\section{Bảng 1}

Thang đo

\begin{tabular}{|c|c|c|c|c|}
\hline \multirow{2}{*}{ Nhân tố } & \multirow{2}{*}{ Mã hóa } & \multicolumn{3}{|c|}{$\begin{array}{l}\text { Thang đo } \\
\end{array}$} \\
\hline & & Nguồn & Số biến sau hiệu chỉnh, ví dụ & Kiểu đo \\
\hline $\begin{array}{l}\text { Sự hài lòng } \\
\text { với việc sử } \\
\text { dụng } \\
\text { PTTTĐT }\end{array}$ & SHL & $\begin{array}{c}\text { Bhattacherjee } \\
\quad(2001)\end{array}$ & $\begin{array}{l}\text { 04 } \\
\text { "Tôi cảm thấy hài lòng với } \\
\text { việc sử dụng PTTTĐT đế cộng } \\
\text { tạc với các thành viên khác } \\
\text { trong đội”... }\end{array}$ & \\
\hline $\begin{array}{l}\text { Thói quen sử } \\
\text { dụng } \\
\text { PTTTĐT }\end{array}$ & TQSD & $\begin{array}{l}\text { Limayem và } \\
\text { Hirt (2003) }\end{array}$ & $\begin{array}{l}05 \\
\text { "Việc sử dụng PTTTĐT để } \\
\text { cộng tác với các thành viên } \\
\text { khác trong đội đã trở thành } \\
\text { thói quen đối với tôi"... }\end{array}$ & $\begin{array}{l}\text { 1: Rất } \\
\text { không } \\
\text { đồng ý -> } \\
\text { 5: Rất đồng }\end{array}$ \\
\hline $\begin{array}{l}\text { Ý định tiếp } \\
\text { tục sử dụng } \\
\text { PTTTĐT }\end{array}$ & YĐTT & $\begin{array}{c}\text { Bhattacherjee } \\
\quad(2001)\end{array}$ & $\begin{array}{l}05 \\
\text { “Tôi dự định tiếp tục sử dụng } \\
\text { PTTTĐT thay vì chấm dứt"... }\end{array}$ & ý \\
\hline $\begin{array}{l}\text { Thành quả } \\
\text { công việc }\end{array}$ & TQCV & $\begin{array}{l}\text { Walumbwa, } \\
\text { Cropanzano, } \\
\text { và Hartnell } \\
(2009)\end{array}$ & $\begin{array}{l}07 \\
\text { "Cho đến thời điểm này, tôi đã } \\
\text { hoàn thành đầy đủ các nhiệm } \\
\text { vụ được giao"... }\end{array}$ & \\
\hline
\end{tabular}




\begin{tabular}{|c|c|c|c|c|}
\hline \multirow{2}{*}{ Nhân tố } & \multirow{2}{*}{ Mã hóa } & \multicolumn{3}{|c|}{ Thang đo } \\
\hline & & Nguồn & Số biến sau hiệu chỉnh, ví dụ & Kiểu đo \\
\hline $\begin{array}{l}\text { Mức độ sử } \\
\text { dụng } \\
\text { PTTTĐT }\end{array}$ & MĐSD & $\begin{array}{l}\text { Bhattacherjee } \\
\text { và Lin (2015) }\end{array}$ & $\begin{array}{l}03 \\
\text { "Việc sử dụng PTTTĐT có thể } \\
\text { giúp tôi hoàn thành ...\% tồng } \\
\text { nội dung công việc hàng ngày } \\
\text { trong đội"... }\end{array}$ & $\begin{array}{c}\text { 1: Từ } 0 \% \\
\text { đến } 20 \% \text {-> } \\
\text { 5: Từ } 81 \% \\
\text { đến } 100 \%\end{array}$ \\
\hline
\end{tabular}

Nguồn: Kết quả phân tích dữ liệu của nhóm nghiên cứu

Nghiên cứu định lượng bằng cách phát bằng bảng câu hỏi theo phương pháp thuận tiện phi xác suất cho các cá nhân đang là thành viên các đội GCPM từ xa tại Việt Nam. Cỡ mẫu 243, đạt yêu cầu về kích thước mẫu tối thiểu (Hair, Black, Babin, \& Anderson, 2014). Dữ liệu được mã hóa, làm sạch và đưa vào phân tích bằng phần mềm SPSS/AMOS qua các bước: (1) thống kê mô tả, (2) kiểm định sơ bộ thang đo bằng phân tích Cronbach's Alpha và EFA, (3) kiểm định mô hình đo lường và mô hình cấu trúc bằng phân tích CFA và SEM.

\section{Kết quả nghiên cứu}

\subsection{Thống kê mô tả}

Có 63 đáp viên đang là trưởng đội $(25,9 \%)$ và 180 đáp viên đang là thành viên $(74,1 \%)$. Có 189 đáp viên đang làm việc trong đội có ít hơn 10 thành viên $(77,8 \%)$ và 54 đáp viên đang làm việc trong đội có từ 10 thành viên trở lên $(22,2 \%)$. Các biến đo lường có giá trị trung bình nằm trong khoảng [3,424; 4,132], độ lệch chuẩn nằm trong khoảng [0,4103; 0,9103].

\subsection{Kiểm định mô hình đo luờng}

\subsubsection{Phân tích nhân tố khám phá}

Kiểm tra độ tin cậy sơ bộ của các thang đo bằng phân tích hệ số Cronbach's Alpha, lần lượt loại 04 biến do |tương quan biến - tổng $<0,3$ và hệ số Cronbach's Alpha của thang đo tăng khi loại biến. Còn 20 biến, phân tích EFA 2 lần (phép quay Promax), lần lượt loại 02 biến do tải lên 02 nhân tố với |hiệu số tải| <0,3, tập dữ liệu còn 18 biến có hệ số tải đều $>0,7$, $\mathrm{KMO}=0,835$ ( $\mathrm{p}=0,000<0,05)$. Có 05 nhân tố hình thành với hệ số Cronbach's Alpha đều >0,7. Tổng phương sai trích $=71,372 \%$ giải thích tương đối tốt sự biến thiên của dữ liệu (Hair et al., 2014) (xem Bảng 2).

\subsubsection{Phân tích nhân tố khẳng định}

Phân tích CFA để kiểm định mô hình đo lường, kết quả cho thấy mô hình đề xuất là phù hợp với dữ liệu, với các chỉ số Chi-square $\left(\chi^{2}\right) / \mathrm{dF}=1,561(<3)(\mathrm{p}=0,000<0,05)$, GFI=0,922 $(>0,9)$, TLI=0,957 $(>0,9), \mathrm{CFI}=0,965(>0,9), \mathrm{IFI}=0,965(>0,9), \mathrm{RMSEA}=0,048(<0,06)$. Hệ số tải chuẩn hóa của 18 biến đều $>0,5(\mathrm{p}=0,000<0,05)$, phương sai trích trung bình (AVE) của các nhân tố đều >0,5 nên thang đo đạt độ hội tụ (Fornell \& Larcker, 1981), ngoài ra hệ số tin cậy tổng hợp $(\mathrm{CR})$ của các nhân tố đều $>0,7$ nên thang đo đạt độ tin cậy (Hair, Hult, Ringle, \& Sarstedt, 2016) (xem Bảng 2). AVE của mỗi nhân tố (trên đường chéo) lớn hơn bình phương hệ số tương quan giữa nhân tố đó với các nhân tố khác nên thang đo đạt độ phân biệt (Hair et al., 2014) (xem Bảng 3). 


\section{Bảng 2}

Kiểm tra độ tin cậy và độ hội tụ của các thang đo

\begin{tabular}{|c|c|c|c|c|c|c|}
\hline Nhân tố & \multicolumn{2}{|c|}{ EFA } & \multicolumn{4}{c|}{ CFA } \\
\hline & $\begin{array}{c}\text { Số biến } \\
\text { sau EFA }\end{array}$ & Hệ số tải & $\begin{array}{c}\text { Số biến } \\
\text { sau CFA }\end{array}$ & $\begin{array}{c}\text { Hệ số tải chuẩn } \\
\text { hóa }\end{array}$ & CR & AVE \\
\hline SHL & 03 & $0,887 \rightarrow 0,896$ & 03 & $0,800 \rightarrow 0,873$ & 0,874 & 0,698 \\
\hline TQSD & 03 & $0,820 \rightarrow 0,831$ & 03 & $0,684 \rightarrow 0,750$ & 0,767 & 0,524 \\
\hline YĐTT & 04 & $0,853 \rightarrow 0,892$ & 04 & $0,793 \rightarrow 0,834$ & 0,892 & 0,673 \\
\hline MĐSD & 03 & $0,838 \rightarrow 0,865$ & 03 & $0,744 \rightarrow 0,788$ & 0,807 & 0,583 \\
\hline TQCV & 05 & $0,743 \rightarrow 0,840$ & 05 & $0,659 \rightarrow 0,811$ & 0,843 & 0,620 \\
\hline
\end{tabular}

Nguồn: Kết quả phân tích dữ liệu của nhóm nghiên cứu

\section{Bảng 3}

Kiểm tra độ phân biệt của các thang đo

\begin{tabular}{|l|c|c|c|c|c|}
\hline \multicolumn{1}{|c|}{ Nhân tố } & SHL & TQSD & YĐTT & MĐSD & TQCV \\
\hline SHL & 0,835 & & & & \\
\hline TQSD & 0,360 & 0,724 & & & \\
\hline YĐTT & 0,384 & 0,315 & 0,820 & & \\
\hline MĐSD & 0,461 & 0,276 & 0,500 & 0,764 & \\
\hline TQCV & 0,322 & 0,353 & 0,304 & 0,360 & 0,787 \\
\hline
\end{tabular}

Nguồn: Kết quả phân tích dữ liệu của nhóm nghiên cứu

\subsection{Kiểm định mô hình cấu trúc}

Phân tích SEM để kiểm định mô hình cấu trúc bằng ước lượng ML, kết quả cho thấy mô hình đạt độ phù hợp chung với các chỉ số Chi- square $(\chi 2) / \mathrm{dF}=1,648(<3)(\mathrm{p}=0,000<0,05)$, $\mathrm{GFI}=0,916(>0,9), \mathrm{TLI}=0,950(>0,9), \mathrm{CFI}=0,958(>0,9), \mathrm{IFI}=0,959(>0,9), \mathrm{RMSEA}=0,052$ $(<0,06)$. Bốn nhân tố $\mathrm{SHL}, \mathrm{TQSD}$, YĐTT và MĐSD giải thích được $16 \%$ phương sai của TQCV (xem Hình 1).

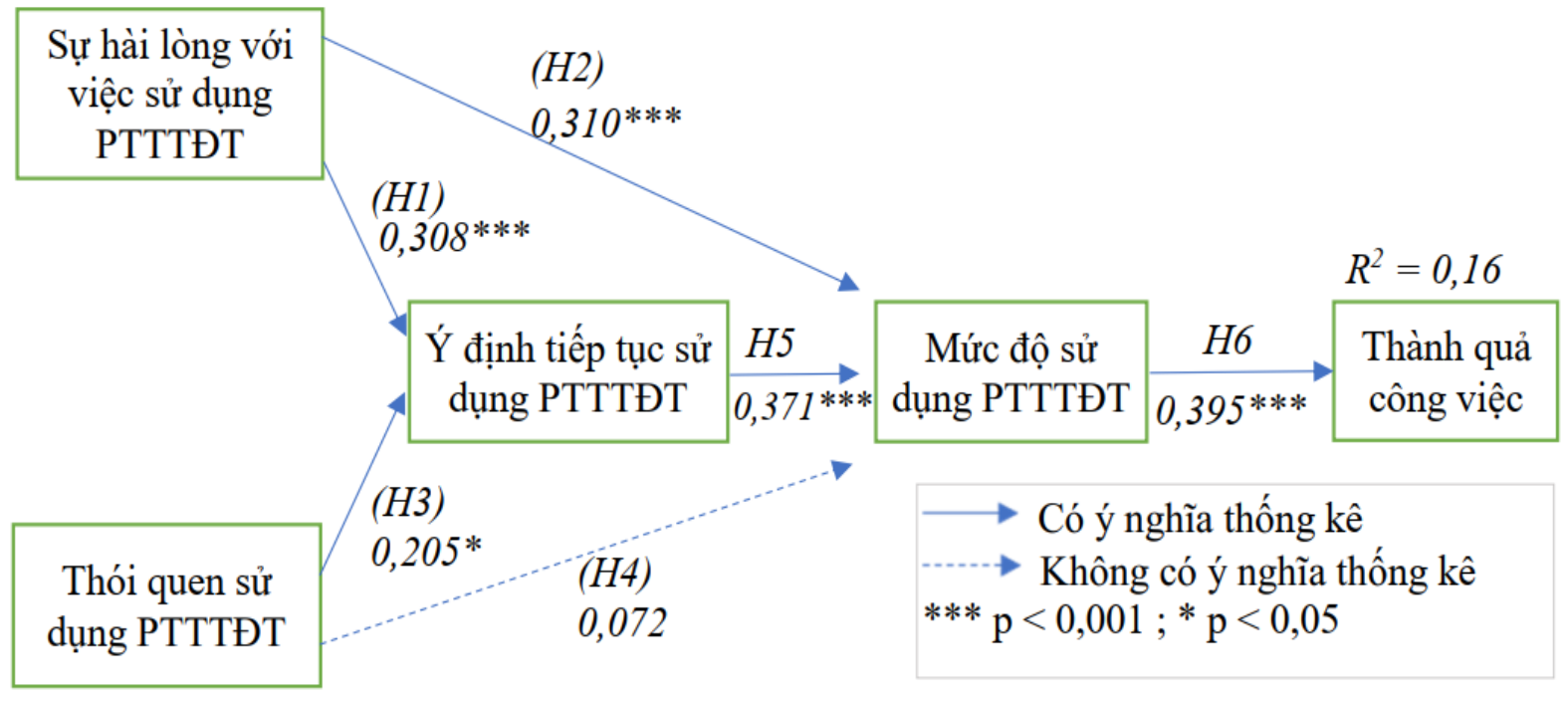

Hình 1. Kết quả phân tích mô hình cấu trúc tuyến tính 
Kiểm tra các mối quan hệ trong mô hình, kết quả cho thấy giả thuyết (H4) bị bác bỏ do mức ý nghĩa $p=0,366>0,05,05 / 06$ giả thuyết còn lại được ủng hộ. SHL và TQSD có ảnh hưởng dương lên YĐTT. SHL và YĐTT có ảnh hưởng dương lên MĐSD. MĐSD có ảnh hưởng dương lên TQCV (Hair et al., 2014) (xem Bảng 4).

\section{Bảng 4}

Kiểm định các giả thuyết

\begin{tabular}{|c|c|c|c|c|}
\hline Mối quan hệ & Giả thuyết & Ước lượng & Mức ý nghĩa & Kết luận \\
\hline SLH -> YĐTT & H1 & 0,308 & $* * *$ & Ung hộ \\
\hline SHL -> MĐSD & H2 & 0,310 & $* * *$ & Ủng hộ \\
\hline TQSD -> YĐTT & H3 & 0,205 & 0,012 & Ung hộ \\
\hline TQSD -> MĐSD & H4 & 0,072 & 0,366 & Bác bỏ \\
\hline YĐTT -> MĐSD & H5 & 0,371 & $* * *$ & Úng hộ \\
\hline MĐSD -> TQCV & H6 & 0,395 & $* * *$ & Ủng hộ \\
\hline
\end{tabular}

Nguồn: Kết quả phân tích dữ liệu của nhóm nghiên cứu

\section{Thảo luận kết quả và kiến nghị}

\section{1. Đóng góp lý thuyết và hàm ý quản trị}

Về mặt lý thuyết, đóng góp của bài báo này là mở rộng và kiểm định lý thuyết tiếp tục sử dụng CNTT/HTTT của Bhattacherjee et al. (2008), với bộ dữ liệu khảo sát ở Việt Nam. Dựa trên mô hình tiếp tục sử dụng CNTT của Bhattacherjee và Lin (2015), bài báo này bổ sung một hậu tố mới (thành quả công việc) và hai mối quan hệ mới $(\mathrm{H} 3$ và H6) và đề xuất một mô hình có cấu trúc mô tả cách thức ảnh hưởng của sự hài lòng với việc sử dụng PTTTĐT, thói quen sử dụng PTTTĐT, ý định tiếp tục sử dụng PTTTĐT, mức độ sử dụng PTTTĐT lên thành quả công việc của thành viên đội GCPM từ xa. Mẫu khảo sát là 243 thành viên các đội GCPM từ xa tại Việt Nam.

Tương tự như Bhattacherjee và Lin (2015), kết quả nghiên cứu của bài báo này cho thấy trong bối cảnh đội GCPM từ xa, cũng tồn tại ảnh hưởng tích cực của sự hài lòng với việc sử dụng PTTTĐT lên ý định tiếp tục sử dụng PTTTĐT $(\beta=0,308)$, ảnh hưởng tích cực của sự hài lòng với việc sử dụng PTTTĐT lên mức độ sử dụng PTTTĐT $(\beta=0,310)$, ảnh hưởng tích cực của ý định tiếp tục sử dụng PTTTĐT lên mức độ sử dụng PTTTĐT $(\beta=0,371)$. Tuy nhiên, so với Bhattacherjee và Lin (2015), bài báo này giúp khám phá thêm ảnh hưởng tích cực của thói quen sử dụng PTTTĐT lên ý định tiếp tục sử dụng PTTTĐT $(\beta=0,205)$, và ảnh hưởng tích cực của mức độ sử dụng PTTTĐT lên thành quả công việc $(\beta=0,395)$. Đồng thời, kết quả nghiên cứu của bài báo này không ủng hộ ảnh hưởng tích cực của thói quen sử dụng PTTTĐT lên mức độ sử dụng PTTTĐT - đây là điểm khác biệt so với Bhattacherjee và Lin (2015) và một số nghiên cứu liên quan (e.g., De Guinea \& Markus, 2009; Kim \& Malhotra, 2005; Limayem \& Hirt, 2003; Wood et al., 2002). Bài báo này bổ sung tài liệu tham khảo về chủ đề tiếp tục sử dụng CNTT và cho thấy vai trò của việc sử dụng công nghệ hỗ trợ đối với kết quả làm việc trong đội ảo - vốn chưa được chú ý nghiên cứu tại Việt Nam.

Về mặt quản trị, từ kết quả nghiên cứu, một vài kiến nghị có thể được đề xuất. Một là, trong đội GCPM từ xa, có một tỉ lệ nhất định $(16 \%)$ sự biến thiên thành quả công việc của thành viên đội có thể được giải thích bởi các yếu tố liên quan đến việc sử dụng PTTTĐT (gồm sự hài lòng với việc sử dụng trước đó, thói quen sử dụng, ý định tiếp tục sử dụng và mức độ sử dụng); 
trong đó, mức độ sử dụng PTTTĐT trực tiếp dẫn đến thành quả công việc. Điều này cho thấy tầm quan trọng của việc sử dụng PTTTĐT đối với kết quả làm việc của thành viên đội trong bối cảnh làm việc gián tiếp thông qua sự hỗ trợ của công nghệ. Hai là, để gia tăng mức độ sử dụng PTTTĐT, có thể tập trung vào việc nâng cao sự hài lòng với việc sử dụng trước đó - tức là khía cạnh cảm xúc, và củng cố ý định tiếp tục sử dụng - tức là khía cạnh nhận thức, đối với việc tiếp tục sử dụng PTTTĐT. Ba là, ý định tiếp tục sử dụng PTTTĐT có thể được củng cố thông qua việc nâng cao sự hài lòng với việc sử dụng trước đó và rèn luyện thói quen sử dụng. Như vậy, cả khía cạnh cảm xúc và khía cạnh nhận thức đối với việc sử dụng PTTTĐT rất đáng được chú ý vì có ảnh hưởng trực tiếp lẫn gián tiếp lên mức độ sử dụng PTTTĐT.

\subsection{Hạn chế và hướng nghiên cứu tiếp theo}

Bài báo này chỉ thực hiện kiểm định mô hình trong một bối cảnh duy nhất là các đội GCPM từ xa tại Việt Nam, vì vậy hướng nghiên cứu kế tiếp có thể là đánh giá lại tính tổng quát của mô hình trong các lĩnh vực khác, và/hoặc có thể tập trung vào việc kiểm định mô hình ở các địa phương hay vùng lãnh thổ khác nhau với các đặc trưng văn hóa riêng. Cuối cùng, dù nhiều học giả cho rằng đội ảo là một cách tổ chức công việc mang tính kỹ thuật - xã hội rõ nét (Carroll \& Wang, 2011; Cogburn, Santuzzi, \& Vasquez, 2011; Painter et al., 2016), tức là giữa các yếu tố liên quan đến việc sử dụng công nghệ hỗ trợ và các yếu tố xã hội có sự tương tác trong quá trình dẫn đến kết quả làm việc trong đội ảo, nhưng ý tưởng đó chưa được thể hiện trong bài báo này, đây có thể là một cơ hội nghiên cứu triển vọng.

\section{Tài liệu tham khảo}

Agudo-Peregrina, Á. F., Hernández-García, Á., \& Pascual-Miguel, F. J. (2014). Behavioral intention, use behavior and the acceptance of electronic learning systems: Differences between higher education and lifelong learning. Computers in Human Behavior, 34, 301314.

Bergiel, B. J., Bergiel, E. B., \& Balsmeier, P. W. (2008). Nature of virtual teams: A summary of their advantages and disadvantages. Management Research News, 31(2), 99-110.

Bhattacherjee, A. (2001). Understanding information systems continuance: An expectationconfirmation model. MIS Quarterly, 25(3), 351-370.

Bhattacherjee, A., \& Lin, C. P. (2015). A unified model of IT continuance: Three complementary perspectives and crossover effects. European Journal of Information Systems, 24(4), 364-373.

Bhattacherjee, A., Perols, J., \& Sanford, C. (2008). Information technology continuance: A theoretic extension and empirical test. Journal of Computer Information Systems, 49(1), $17-26$.

Burton-Jones, A., \& Gallivan, M. J. (2007). Toward a deeper understanding of system usage in organizations: A multilevel perspective. MIS Quarterly, 31(4), 657-679.

Burton-Jones, A., \& Straub, D. W., Jr. (2006). Reconceptualizing system usage: An approach and empirical test. Information systems research, 17(3), 228-246. 
Carroll, J. M., \& Wang, J. (2011). Designing effective virtual organizations as sociotechnical systems. Paper presented at the Hawaii International Conference on System Sciences, USA.

Case, T., Cuellar, M., \& Tabatabaei, M. (2015). The intention to re-adopt collaboration and communication technologies by project teams. Paper presented at the Americas Conference on Information Systems, San Juan, Puerto Rico.

Chaves, M. S., de Araújo, C. C. S., Teixeira, L., Rosa, D., Júnior, I., \& Nogueira, C. (2016). A new approach to managing Lessons Learned in PMBoK process groups: The Ballistic 2.0 Model. International journal of information systems and project management, 4(1), 2745 .

Cogburn, D. L., Santuzzi, A., \& Vasquez, F. K. E. (2011). Developing and validating a sociotechnical model for geographically distributed collaboration in global virtual teams. Paper presented at the Hawaii International Conference on System Sciences, USA.

Davis, G., Ein-Dor, P. R., King, W., \& Torkzadeh, R. (2006). IT offshoring: History, prospects and challenges. Journal of the Association for Information Systems, 7(11).

De Guinea, A. O., \& Markus, M. L. (2009). Why break the habit of a lifetime? Rethinking the roles of intention, habit, and emotion in continuing information technology use. MIS Quarterly, 33(3), 433-444.

Doong, H.-S., \& Lai, H. (2008). Exploring usage continuance of e-negotiation systems: Expectation and disconfirmation approach. Group Decision and Negotiation, 17(2), 111126.

Dube, S., \& Marnewick, C. (2016). A conceptual model to improve performance in virtual teams. South African Journal of Information Management, 18(1), 1-10.

Dulebohn, J. H., \& Hoch, J. E. (2017). Virtual teams in organizations. Human Resource Management Review, 27(4), 569-574.

Ebrahim, N., Ahmed, S., \& Taha, Z. (2011). Virtual teams and management challenges. Academic Leadership: The Online Journal, 9(3).

Fornell, C., \& Larcker, D. F. (1981). Evaluating Structural equation models with unobservable variables and measurement error. Journal of Marketing Research, 18(1), 39-50.

Friedrich, R. (2017). The virtual team maturity model: Performance improvement of virtual teams. Berlin, Germany: Springer.

Gefen, D. (2003). TAM or just plain habit: A look at experienced online shoppers. Journal of Organizational and End User Computing (JOEUC), 15(3), 1-13.

Gilani, S., Iranmanesh, M., Nikbin, D., \& Zailani, S. (2016). EMR continuance usage intention of healthcare professionals. Informatics for Health and Social Care, 42(2), 153-165.

Godin, J., Leader, L., Gibson, N., Marshall, B., Poddar, A., \& Cardon, P. W. (2017). Virtual teamwork training: Factors influencing the acceptance of collaboration technology. International Journal of Information and Communication Technology, 10(1), 5-23. 
Greer, B. M., Luethge, D. J., \& Robinson, G. (2017). Utilizing virtual technology as a tool to enhance the workforce diversity learning. In Discrimination and diversity: Concepts, methodologies, tools, and applications (pp. 822-843).

Griffith, T. L., Sawyer, J. E., \& Neale, M. A. (2003). Virtualness and knowledge in teams: Managing the love triangle in organizations, individuals, and information technology. MIS Quarterly, 27, 265-287.

Hair, J. F., Black, W. C., Babin, B. J., \& Anderson, R. E. (2014). Multivariate data analysis: Pearson new international edition. London, UK: Pearson Education Limited.

Hair, J. F., Hult, G. T. M., Ringle, C., \& Sarstedt, M. (2016). A primer on partial least squares structural equation modeling (PLS-SEM). Thousand Oaks, CA: SAGE.

Huang, T. C.-K., Wu, L., \& Chou, C.-C. (2013). Investigating use continuance of data mining tools. International Journal of Information Management, 33(5), 791-801.

Huynh, C. T. M., Nguyen, T. M., \& Hang, P. L. C. (2017). Knowledge sharing in virtual teams: A research in information technology companies in Vietnam. Journal of Science Ho Chi Minh City Open University, 21(1), 75-88.

Jasperson, J. S., Carter, P. E., \& Zmud, R. W. (2005). A comprehensive conceptualization of post-adoptive behaviors associated with information technology enabled work systems. MIS Quarterly, 29(3), 525-557.

Kayworth, T. R., \& Leidner, D. E. (2002). Leadership effectiveness in global virtual teams. Journal of Management Information Systems, 18(3), 7-41.

Kim, B. (2012). The diffusion of mobile data services and applications: Exploring the role of habit and its antecedents. Telecommunications Policy, 36(1), 69-81.

Kim, S. S., \& Malhotra, N. K. (2005). A longitudinal model of continued IS use: An integrative view of four mechanisms underlying post-adoption phenomena. Management Science, 51(5), 741-755.

Lian, J.-W., Yen, D. C., \& Wang, Y.-T. (2014). An exploratory study to understand the critical factors affecting the decision to adopt cloud computing in Taiwan hospital. International Journal of Information Management, 34(1), 28-36.

Limayem, M., \& Cheung, C. M. (2008). Understanding information systems continuance: The case of Internet-based learning technologies. Information \& Management, 45(4), 227 232.

Limayem, M., \& Hirt, S. G. (2003). Force of habit and information systems usage: Theory and initial validation. Journal of the Association for Information Systems, 4(1).

Lipnack, J., \& Stamps, J. (2000). Virtual teams: People working across boundaries with technology (2nd ed.). Hoboken, NJ: Wiley.

Ng, S. Y., Ching, S. Y., Chung, Y. T., \& Dee, C. Y. (2016). Determinants of continuance usage intention of social network services in Malaysia. Retrieved October, 22, 2018, from http://eprints.utar.edu.my/2015/ 
Ocker, R. J., \& Fjermestad, J. (2008). Communication differences in virtual design teams: Findings from a multi-method analysis of high and low performing experimental teams. DATA BASE for Advances in Information Systems, 39, 51-67.

Ozcelik, Y. (2010). The rise of teleworking in the USA: Key issues for managers in the information age. International Journal of Business Information Systems, 5(3), 211-229.

Painter, G., Posey, P., Austrom, D., Tenkasi, R., Barrett, B., \& Merck, B. (2016). Sociotechnical systems design: Coordination of virtual teamwork in innovation. Team Performance Management, 22(7/8), 354-369.

Piguing, A., \& Ko, I. (2016). Continuance intention to use social network game: The Philipines case. Paper presented at the Hawaii International Conference on System Sciences, USA.

Robinson, M., \& Kalakota, R. (2004). Offshore outsourcing: Business models, ROI and best practices (2nd ed.). Milano, Italy: Mivar Pr Inc.

Sahay, S., Nicholson, B., \& Krishna, S. (2003). Global IT outsourcing: Software development across borders. Cambridge, UK: Cambridge University Press.

Santillan, C., \& Horwitz, S. K. (2016). Application of collaboration technology to manage diversity in global virtual teams: The thinklet-based CE approach. In Handbook of research on race, gender, and the fight for equality (pp. 240-266).

Scheier, M. F., \& Carver, C. S. (1982). Self-consciousness, outcome expectancy, and persistence. Journal of Research in Personality, 16(4), 409-418.

Shanmugam, M., Jusoh, Y. Y., Nor, R. N. H., \& Jabar, M. A. (2015). A theoretical extension and empirical investigation for continuance use in social networking sites. Arpn Journal of Engineering and Applied Sciences, 10(23), 17730-17739.

Shiue, Y.-M., \& Hsu, Y.-C. (2017). Understanding factors that affecting continuance usage intention of game-based learning in the context of collaborative learning. Eurasia Journal of Mathematics, Science and Technology Education, 13(10), 6445-6455.

Solomon, C. M. (2016). Trends in global virtual teams. Retrieved October, 25, 2018, from https://www.rw-3.com/blog/trends-in-global-virtual-teams

Sun, Y., Bhattacherjee, A., \& Ma, Q. (2009). Extending technology usage to work settings: The role of perceived work compatibility in ERP implementation. Information \& Management, 46(6), 351-356.

Thakur, R. H., Summey, J., \& John, J. (2013). A perceptual approach to understanding usergenerated media behavior. Journal of Consumer Marketing, 30(1), 4-16.

Tran Ngoc (2018). Forbes: Việt Nam sớm thành trung tâm gia công phần mềm của thế giới [Forbes: Vietnam soon became the world's outsourcing center]. Retrieved October, 25, 2018, from VOV website: https://vov.vn/kinh-te/forbes-viet-nam-som-thanh-trung-tamgia-cong-phan-mem-cua-the-gioi-768755.vov

Van Anh (2019). Xuất khẩu phần mềm Việt Nam năm 2018 uớc đạt 3,5 tỷ USD doanh thu [Vietnam's software export in 2018 is estimated at 3.5 billion USD in revenue]. Retrieved 
October, 20, 2018, from Ictnews website: https://ictnews.vn/cntt/xuat-khau-phan-memviet-nam-nam-2018-uoc-dat-3-5-ty-usd-doanh-thu-177818.ict

Walumbwa, F. O., Cropanzano, R., \& Hartnell, C. A. (2009). Organizational justice, voluntary learning behavior, and job performance: A test of the mediating effects of identification and leader member exchange. Journal of Organizational Behavior, 30(8), 1103-1126.

Wise, T. P. (2016). Trust in virtual teams: Organization, strategies and assurance for successful projects. Boca Raton, FL: CRC Press.

Wood, W., Quinn, J. M., \& Kashy, D. A. (2002). Habits in everyday life: Thought, emotion, and action. Journal of Personality and Social Psychology, 83(6), 1281-1297. 